“ (C) 2016 IEEE. Personal use of this material is permitted. Permission from IEEE must be obtained for all other uses, in any current or future media, including

reprinting/republishing this material for advertising or promotional purposes, creating new collective works, for resale or redistribution to servers or lists, or reuse of any copyrighted component of this work in other works." 


\title{
A Wideband Polarization Reconfigurable Antenna for WLAN Applications
}

\author{
Lu-Yang Ji ${ }^{1}$, Pei-Yuan Qin ${ }^{2}$, Y.Jay Guo ${ }^{2}$, Guang Fu${ }^{1}$, Raj Mittra ${ }^{3}$ \\ ${ }^{1}$ School of Electronic Engineering, Xidian University, Xi'an, China, luyangji078@gmail.com \\ ${ }^{2}$ Faculty of Engineering and IT, UTS, Sydney, Australia, Peiyuan.Qin@uts.edu.au \\ ${ }^{3}$ University of Central Florida, USA, and King Abdulaziz University, Saudi Arabia, rajmittra@ieee.org
}

\begin{abstract}
This paper proposes a wideband polarization reconfigurable antenna design for WLAN applications. It consists of a shorted annular patch (SAP) antenna as the source, a partially reflective surface (PRS) structure to enhance the gain, and a reconfigurable Wilkinson power divider as the feed network. The antenna can electronically alter its polarization between linear polarization (LP), left-hand circular polarization (LHCP), and right-hand circular polarization (RHCP), achieving an overlapped $10 \mathrm{~dB}$ impedance bandwidth and $3 \mathrm{~dB}$ axial-ratio bandwidth of 4.68-5.33 GHz (13\%), thus outperforming most of the reported polarization reconfigurable antennas in terms of the frequency bandwidth.
\end{abstract}

Index Terms-polarization reconfigurable antenna, reconfigutable feed network.

\section{INTRODUCTION}

In the last two decades, polarization reconfigurable antennas have attracted much interest in the wireless communication systems owing to their advantages of mitigating signal fading in multipath propagation environments and realizing double transmission channels for frequency-reuse transceivers [1]. Many polarization reconfigurable antennas have been designed that can switch between two orthogonal liner polarizations or two different circular polarization modes. However, relatively few antennas have been proposed to realize the switch between LP and two CPs because it is difficult to simultaneously achieve a good impedance match for both the LP and CP. Some work has been done to address the above problem by making the antenna more versatile. In [2], four pin-diodes were used on a corner-truncated square patch to produce LP and CP radiation with a small impedance bandwidth (2.5\%). In [3], a U-slot microstrip patch antenna was proposed that can provide polarization switch between LP and CP. However, the overlapped operating frequency bandwidth is less than $6.1 \%$. A square-ring microstrip antenna excited by a $\mathrm{T}$-junction power divider and a pair of varactor diodes has been reported in [4]. It enables the polarization to be tuned among LP and two CPs across a bandwidth of $1.4 \%$ around $2 \mathrm{GHz}$. In [5], a branch line coupler with four microstrip feeding lines is employed as the feed network for an aperture-fed square patch antenna. Eight PIN diodes are inserted in the feed network to provide quadpolarization reconfigurability, in two orthogonal linear polarizations and two orthogonal circular polarizations. The impedance bandwidth achieved in this design is $3.67 \%$ at 2.45 $\mathrm{GHz}$. A traditional annular slot antenna can achieve a wider bandwidth of $4.7 \%$ [6] by employing an arrow-shaped coupling strip with a PIN diode inserted in each arm. In summary, it has been found that most of the reported antennas can switch their polarizations in LP and two CP modes across an overlapped impedance bandwidth less than $10 \%$. As the wideband communication is commonplace for recent wireless communication systems, a wideband polarization reconfigurable antenna is highly desirable.

A wideband polarization reconfigurable PRS antenna, which employs a SAP antenna as the source and a reconfigurable Wilkinson power divider network, is proposed in this work to realize polarization switching among LP, LHCP, and RHCP, with an overlapped $10 \mathrm{~dB}$ impedance bandwidth and $3 \mathrm{~dB}$ axial-ratio bandwidth of $4.68-5.33 \mathrm{GHz}(13 \%)$. The reconfigurable feed network utilizes the conventional Wilkinson power divider and achieves a phase difference of $0^{\circ}$ or $\pm 90^{\circ}$ between the two output ports across a relatively wide frequency range by incorporating two phase shifters and eight PIN-diodes into the power divider, a phase difference of $0^{\circ}$ or $\pm 90^{\circ}$ between the two output ports can be achieved across a relatively wide frequency range. The network is highly integrated in a way such that it does not require any additional impedance matching networks, hence the structure is compact.

\section{RECONFIGURABLE FEED NETWORK}

The configuration of the network is shown in Fig.1. It is based on a reformative Wilkinson power divider with a phase shifter inserted in each branch. The details of the phase shifter are shown in the inset of Fig. 1 and its dimensions are listed in Table I. The phase shifter is comprised of two separate microstrip lines (a short one and a long one as shown in Fig.1) and each line has two gaps etched at the edges for inserting PIN diodes. The long microstrip lines are approximately $\lambda_{g} / 4$ longer than the short microstrip lines, where $\lambda g$ is the guided wavelength at the resonant frequency. By switching the diodes on and off, the length of each branch of the power divider can be varied, which produces the phase difference between the two output ports and, in turn, realizes polarization reconfigurability. Four groups of PIN diodes are used in this design (A and A', B and B', C and C', D and D'). When either the diode groups A and $\mathrm{D}$ are switched on and the others are off, or when groups $\mathrm{B}$ and $\mathrm{C}$ are switched on and the others are off, no phase difference exists between the two branches. These two states are referred to as $\mathrm{S} 1$. When the diodes $\mathrm{A}, \mathrm{A}^{\prime}$ and $\mathrm{C}, \mathrm{C}^{\prime}$ are switched on, and the others are switched off, the left branch generates a $90^{\circ}$ delay 
to the right one, which is called S2; When the diodes $\mathrm{B}, \mathrm{B}$ ' and D, D' are switched on with the others off, a $90^{\circ}$ phase lead can be produced at the left branch and this state is referred to as S3. The realized phase differences from the network can be used in a reconfigurable antenna design to realize polarization switching among LP, RHCP and LHCP.

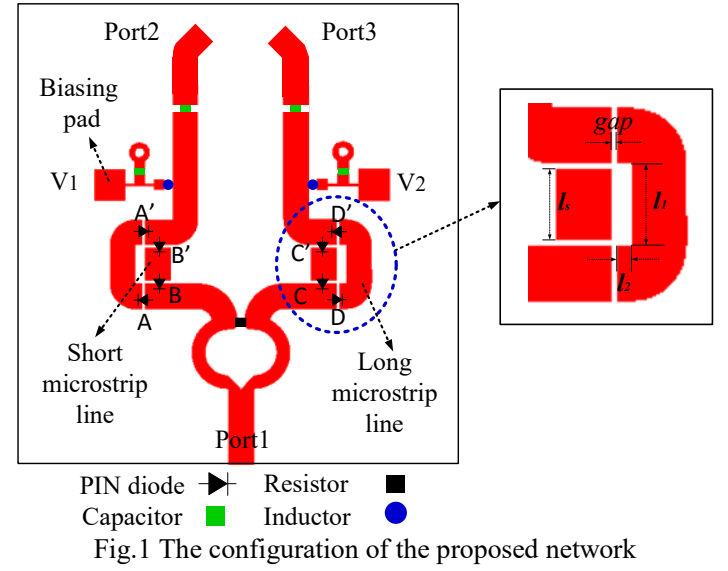

TABLE I

\begin{tabular}{|c|c|c|c|c|}
\hline Parameter & $l_{1}$ & $l_{2}$ & $l_{s}$ & gap \\
\hline Value $(\mathrm{mm})$ & 5.5 & 0.7 & 4.8 & 0.35 \\
\hline
\end{tabular}

In order to verify the operation of the proposed network, a prototype has been designed on an $80 \mathrm{~mm} \times 50 \mathrm{~mm} \times 1.524 \mathrm{~mm}$ Rogers4003 substrate and has been simulated. Based on the PIN diode datasheet of MA4FCP300, the diode is modelled as a series circuit consisting of a $4 \Omega$ resistor and a $0.6 \mathrm{nH}$ inductor for the on-state and a parallel circuit consisting of a capacitor of $0.04 \mathrm{pF}$ and a resistor of about $20 \mathrm{k} \Omega$ for the off-state. The biasing network is also shown in Fig. 1. The PIN diodes on the two short microstrip lines are oriented in the same directions, and those on the two long microstrip lines are placed in the opposite directions. A capacitor is inserted on the top part of each branch not only to realize RF continuity, but to provide DC isolation for the two biasing voltages on the two branches. When $\mathrm{V} 1$ and V2 are connected together and applied to a negative voltage relative to Port- 1 that is connected to the ground, the diodes group A and group D on the two long lines are switched on, which results in a $0^{\circ}$ phase difference between Port- 2 and Port- 3 and leads to a linear polarization. A $90^{\circ}$ phase difference between Port-2 and Port-3 can be achieved by applying a positive voltage to $\mathrm{V} 1$ and grounding V2to obtain a righthanded circular polarization, and vice versa.

Here, we only study the magnitude of the input reflection coefficient for Port-1 $\left(\left|S_{11}\right|\right)$ and the phase difference $\varphi_{23}$ between Port-2 and Port-3. It is observed from Fig.2 that $\left|S_{11}\right|$ is below $10 \mathrm{~dB}$ across the frequency band ranging from $5 \mathrm{GHz}$ to $6 \mathrm{GHz}$ for all the three states. Furthermore, $\varphi_{23}$ values for the three states $\left(\mathrm{S} 1, \mathrm{~S} 2\right.$ and S3) remain approximately $0^{\circ},-90^{\circ}$, and $90^{\circ}$, respectively, in this frequency band.

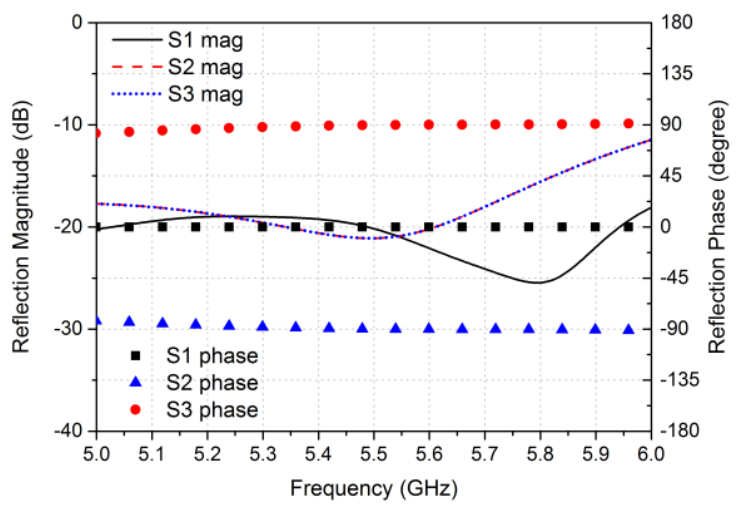

Fig.2 Simulated results of the proposed network

\section{PRS ANTENNA DESIGN}

Fig. 3 shows the configuration of the proposed polarization reconfigurable PRS antenna. It consists of a PRS structure on top, an SAP antenna as the source located above the ground plane, and the proposed feed network underneath the ground plane. The dimensions of the entire antenna are $110 \mathrm{~mm} \times 110$ $\mathrm{mm}(1.83 \lambda \times 1.83 \lambda$ at $5 \mathrm{GHz})$. The PRS material is constructed from a $0.8 \mathrm{~mm}$ thick FR4 substrate and consists of an array of 5 $\times 5$ square metallic patches (SMP). The length of the patch cells $\left(P_{a}\right)$ is $18 \mathrm{~mm}$ and the periodicity between two adjacent patches (P) is $24 \mathrm{~mm}$, as shown in the inset of Fig.3. The cavity height $\left(L_{r}\right)$ is set to be $32 \mathrm{~mm}$.

The source is a two-layer structure fed by two probes with a diameter of $1.3 \mathrm{~mm}$. For the first layer, an SAP antenna is etched on the upper side of a 1.524-mm-thick Rogers 4003 substrate. The diameter of the annular patch $\left(D_{a}\right)$ is $24.6 \mathrm{~mm}$. The patch is shorted to the ground by plating a metallic via hole with a diameter of $11.6 \mathrm{~mm}\left(D_{b}\right)$. The metal of the other side of the substrate serves as the ground plane. For the second layer, the feed network is printed on one side of another 1.524-mm-thick Rogers4003 substrate and the metal of the other side of this substrate is etched off. The SAP antenna and the feed network are connected by two metal probes with a diameter of $1.3 \mathrm{~mm}$. The probes are located at $7.4 \mathrm{~mm}\left(L_{f}\right)$ from the center of the SAP antenna and are sequentially rotated by $90^{\circ}$. The probes are isolated from the ground by cutting two circular slots, with a radius of $1 \mathrm{~mm}$ around them.

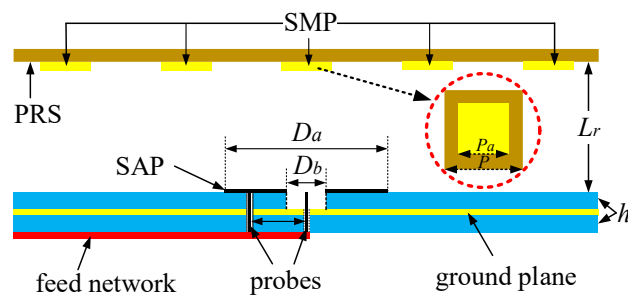

Fig.3 Schematics of the proposed polarization reconfigurable PRS structure

\section{ANTENNA RESULTS}

Figs. 4 show that the simulated $10 \mathrm{~dB}$ impedance bandwidth can cover a frequency band from $4.65 \mathrm{GHz}$ to $5.33 \mathrm{GHz}$ for LP and from $4.63 \mathrm{GHz}$ to $5.84 \mathrm{GHz}$ for LHCP and RHCP. Fig. 5 
gives the simulated axial ratios for the $\mathrm{CP}$ modes. It can be seen that from $4.68 \mathrm{GHz}$ to $5.46 \mathrm{GHz}(15.4 \%)$ the axial ratio is below $3 \mathrm{~dB}$ for both LHCP and RHCP. Considering the overlapped 10 $\mathrm{dB}$ impedance bandwidth of the three polarizations and the $3 \mathrm{~dB}$ axial ratio bandwidth, the overlapped frequency bandwidth ranges from $4.68 \mathrm{GHz}$ to $5.33 \mathrm{GHz}(13 \%)$ for realizing polarization switching between LP and two CP modes. Fig.6 shows the simulated realized gains for the three polarizations. The results for $\mathrm{LP}$ ranges from $7.5 \mathrm{dBi}$ to $11.3 \mathrm{dBi}$ while the results for $\mathrm{CP}$ ranges from $7.3 \mathrm{dBic}$ to $11.2 \mathrm{dBic}$.

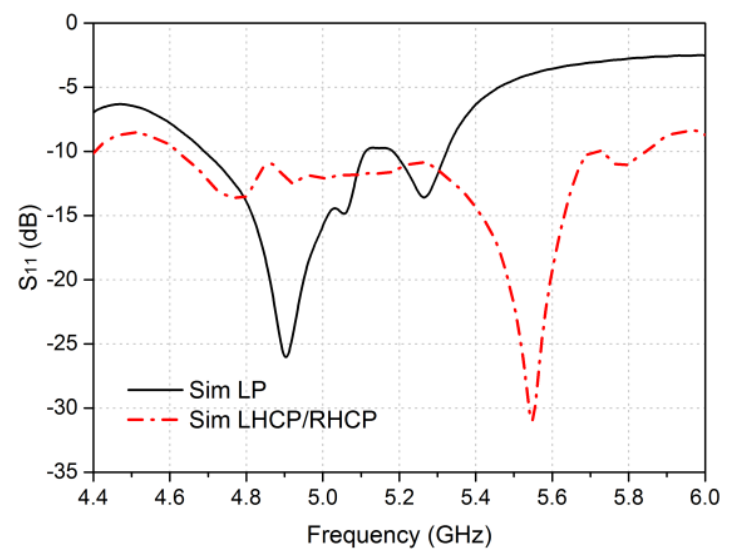

Fig.4 Input reflection coefficients of the proposed antenna for LP and CP

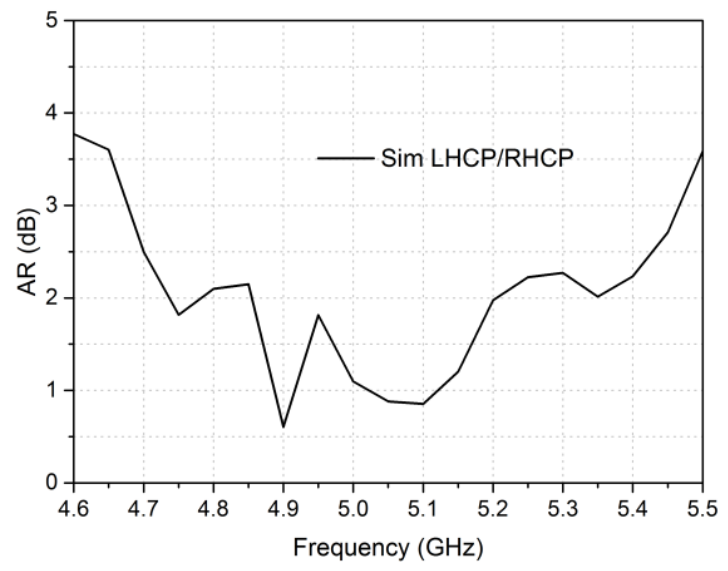

Fig.5 Axial ratio of the proposed antenna

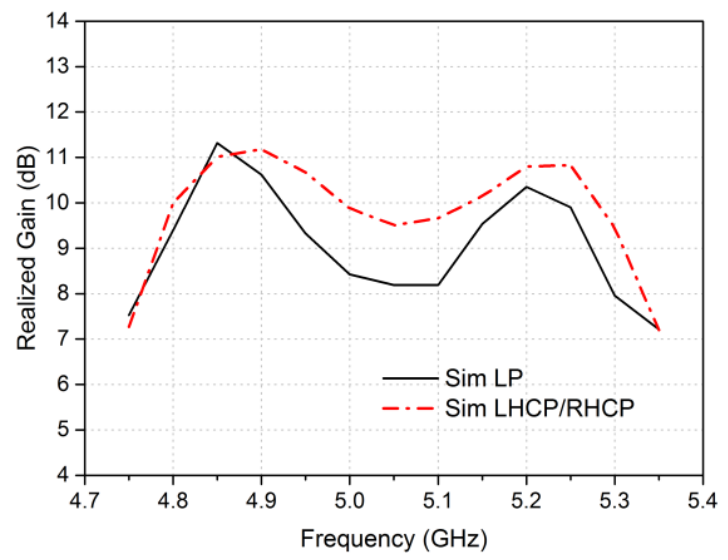

Fig.6 Realized gain of the proposed antenna

\section{CONCLUSION}

A wideband polarization reconfigurable PRS antenna has been proposed in this work. By controlling four PIN diodes embedded in each branch of a Wilkinson power divider, the antenna can be reconfigured to generate LP, RHCP, and LHCP radiation patterns. Compared to most of the reported designs, a wider overlapped frequency range ( $13 \%$ impedance bandwidth) has been realized. The measured radiation patterns and realized gains will be included in the presentation.

\section{REFERENCES}

[1] P. Y. Qin, Y. J. Guo, and C. Ding, "Dual-Band Polarization Reconfigurable Antenna for WLAN Systems," IEEE Trans. Antennas Propag., vol. 61, no. 11, pp.5706-5713, Nov. 2013J. Clerk Maxwell, A Treatise on Electricity and Magnetism, 3rd ed., vol. 2. Oxford: Clarendon, 1892, pp.68-73.

[2] Y. J. Sung, T. U. Jang, and Y. S. Kim, "A reconfigurable microstrip antenna for switchable polarization," IEEE Microw. Wireless Compon.Lett., vol. 14, no. 11, pp. 534-536, Nov. 2004.

[3] P. Y. Qin, A. R. Weily, Y. J. Guo, and C. H. Liang, "Polarization reconfigurable U-Slot ppatch antenna," IEEE Trans. Antennas Propag., vol. 58 , no. 10 , pp. 3383-3388, Oct. 2010.

[4] J. -F. Tsai and J. -S. Row, "Reconfigurable square-ring microstrip antenna," IEEE Trans. Antennas Propag., vol. 61, no. 5, pp. 2857-2860, May 2013.

[5] Y.-F. Wu, C.-H. Wu, D.-Y. Lai, and F.-C. Chen, "A reconfigurable quadri-polarization diversity aperture-coupled patch antenna," IEEE Trans. Antennas Propag., vol. 55, no. 3, pp. 1009-1012, Mar. 2007.

[6] J. -S. Row, W. -L. Liu, and T. -R. Chen, "Circular polarization and polarization reconfigurable designs for annular slot antennas," IEEE Trans. Antennas Propag., vol. 60, no. 12, pp. 5998-6002, Dec. 2012. 